\title{
Sync for Genes: Making Clinical Genomics Available for Precision Medicine at the Point-of-Care
}

\author{
Stephanie J. Garcia ${ }^{1}$ Teresa Zayas-Cabán ${ }^{1}$ Robert R. Freimuth ${ }^{2}$ \\ 1 Office of the National Coordinator for Health Information \\ Technology, Washington, District of Columbia, United States \\ 2 Division of Digital Health Sciences, Department of Health Sciences \\ Research, Mayo Clinic, Rochester, Minnesota, United States \\ Address for correspondence Stephanie J. Garcia, MPH, Office of the \\ National Coordinator for Health Information Technology (ONC), U.S. \\ Department of Health and Human Services, 330 C Street SW, Floor 7 , \\ Washington, DC 20024, United States \\ (e-mail: Stephanie.Garcia@hhs.gov). \\ Appl Clin Inform 2020;11:295-302.
}

\begin{abstract}
Keywords

- genetics

- implementation and deployment

- precision medicine

- electronic health records and systems

- Fast Healthcare Interoperability Resources

- Health Level Seven International

- standards adoption

Background Making genomic data available at the point-of-care and for research is critical for the success of the Precision Medicine Initiative (PMI), a research initiative which seeks to change health care by "tak(ing) into account individual differences in people's genes, environments, and lifestyles." The Office of the National Coordinator for Health Information Technology (ONC) led Sync for Genes, a program to develop standards that make genomic data available when and where it matters most. This article discusses lessons learned from recent Sync for Genes activities.

Objectives The goals of Sync for Genes were to (1) demonstrate exchange of genomic data using health data standards, (2) provide feedback for refinement of health data standards, and (3) synthesize project experiences to support the integration of genomic data at the point-of-care and for research.

Methods Four organizations participated in a program to test the Health Level Seven International $\left(\mathrm{HL} 7^{\circledR}\right)$ Fast Healthcare Interoperability Resources $\left(\mathrm{FHIR}{ }^{\circledR}\right)$ standard, which supports sharing genomic data. ONC provided access to subject matter experts, resources, tools, and technical guidance to support testing activities. Three of the four organizations participated in HL7 FHIR Connectathons to test FHIR's ability to exchange genomic diagnostic reports.

Results The organizations successfully demonstrated exchange of genomic diagnostic reports using FHIR. The feedback and artifacts that resulted from these activities were shared with HL7 and made publicly available. Four areas were identified as important considerations for similar projects: (1) FHIR proficiency, (2) developer support, (3) project scope, and (4) bridging health information technology and genomic expertise.

Conclusion Precision medicine is a rapidly evolving field, and there is opportunity to continue maturing health data standards for the exchange of necessary genomic data, increasing the likelihood that the standard supports the needs of users.
\end{abstract}

\section{Background and Significance}

The Precision Medicine Initiative (PMI), launched in 2015, ignited several projects across federal government to lead the way for the nation to improve the effectiveness of care delivery by moving away from a "one-size-fits-all" approach. ${ }^{1}$ The All of Us Research Program (All of Us) led by the National
Institutes of Health $(\mathrm{NIH})$ is a leading example of these projects. $^{2}$ All of Us is building a cohort of one million people who are donating their health data, including genomic data, to speed up the rate at which health research can lead to medical breakthroughs. ${ }^{3}$ In a related effort, the National Cancer Institute is advancing precision oncology by "expanding precision medicine clinical trials, overcoming drug resistance, received

August 27, 2019

accepted after revision

February 4, 2020 (c) 2020 Georg Thieme Verlag KG Stuttgart · New York
DOI https://doi.org/

10.1055/s-0040-1708051. ISSN 1869-0327. 
developing new laboratory models for research, and developing a national cancer knowledge system." ${ }^{4}$ The Food and Drug Administration (FDA) is developing new regulatory approaches for evaluating next-generation genomic sequencing technologies and launched precision FDA a crowd-sourced, cloud-based platform where the community can test, develop, and validate next-generation sequencing (NGS) software and methods. ${ }^{5}$ The Office of the National Coordinator for Health Information Technology (ONC) is participating in the PMI by accelerating opportunities for innovative collaboration through pilot testing of standards that support health information technology (IT) interoperability for research, encouraging adoption of policies and standards to support privacy and security, and advancing standards that support a participant-driven approach to patient data contribution. ${ }^{6}$

Subsequently, other federal agencies joined the PMI including the Office for Civil Rights, Veterans Affairs, Department of Defense, and others. Most recently, the Health Resources and Services Administration is supporting community health center participation in the All of Us Research Program. ${ }^{7}$ The 21st Century Cures Act legislatively established the roles of NIH, FDA, and ONC in leading the nation toward broadly available precision medicine. ${ }^{8}$ Collectively, the PMI-related programs and this legislation chart the path for the development and implementation of innovative technologies that can realize precision medicine nationwide.

The concept of precision medicine has a long history, and its scope has expanded to include new data types as technology has evolved to collect and analyze increasing information about how we live, work, and play. ${ }^{9,10}$ A critical component of precision medicine is genomic data. Technological advances in genomic testing have brought the possibility of precision medicine at the molecular level to the present. ${ }^{11}$ There have been exciting developments in precision medicine as genomic data have successfully been incorporated into the provision of care for certain use cases. ${ }^{12}$ This success may encourage health care providers to implement precision medicine to keep pace with the evolving science and consumer expectations for better care and tailored treatment. To facilitate the use of genomic data at the point-of-care and for research, it must be available in standardized, computable formats, which is currently not common practice. ${ }^{13}$ Recent work has found inconsistences in how genomic information is shared and delivered at the point-of-care, which may make it more difficult for clinicians to interpret and use that information in clinical practice. ${ }^{14}$ Some efforts have been made to harmonize relevant data needed for precision medicine research creating an imperative for standards development and testing in this field. ${ }^{15}$

The Health Level Seven International (HL7) Fast Healthcare Interoperability Resources (FHIR) standard is considered a foundational health information standard for the interoperable exchange of electronic health data. ${ }^{16}$ FHIR leverages application programming interfaces (APIs) to securely share discrete, structured data rather than unstructured documents that are difficult to parse. FHIR has shown promise in enabling standardized exchange of relevant clinical information in other use cases such as family planning and reproductive health, and effective matching of patients to platelets. ${ }^{17,18}$ Effective use of standards such as FHIR, however, requires accurately reflecting the needs of relevant clinical use cases, such as sharing all clinical data needed to interpret genomic information and deliver precision medicine. ${ }^{19}$ Testing standards as they are being developed is critical to inform their development and help ensure they meet user needs.

ONC launched Sync for Genes in 2016 as part of the PMI at a time of rapid advancements in interoperability standards for health IT. ONC designed Sync for Genes to advance development and testing of standards that support the sharing of genomic data. Sync for Genes used a cohort model to facilitate knowledge sharing, which is critical in emerging fields and domains. Sync for Genes organizations tested mature and emerging health IT standards, including the FHIR standard, and identified critical elements and challenges that need to be overcome to make actionable genomic data available electronically when and where it matters most. ${ }^{20}$

The findings and results from the first phase of Sync for Genes (Phase 1), which concluded in 2017, contributed to the development of the FHIR Clinical Genomics Implementation Guide and the Clinical Genomics Domain Analysis Model, which are artifacts that support the implementation of the FHIR standard for the exchange of clinical genomics data. ${ }^{20,21}$ Phase 1 explored the ability to use FHIR for querying, how to define and use value sets within FHIR, and considerations when using contained FHIR resources. Phase 1 identified several areas for future focus, which informed the next steps for Sync for Genes (referred to here as Phase 2). This included the testing of additional use cases and demonstrating data exchange among multiple stakeholders. This article describes the approach and findings from Phase 2, which ended in March 2019, focusing on lessons learned that organizations may find helpful when making genomic data available at the point-of-care.

\section{Objectives}

The goal of Phase 2 was to demonstrate exchange of genomic data using health data standards by engaging four organizations to test the FHIR Clinical Genomics specification for specific use cases. These individual projects and related activities were designed to yield feedback that could enhance the continued development of health data standards, specifically the FHIR Clinical Genomics specification. A secondary goal was to synthesize the experiences of these organizations into lessons learned that could be applicable to others that are interested in using data standards to make clinical genomic data available at the point-of-care and for research.

\section{Methods}

To meet the objective of demonstrating the electronic exchange of genomic data, potential participants in Phase 2 needed to demonstrate technical capacity to test standards-based sharing of genomic data and committed resources during the Phase 2 project period. The organizations that were accepted to participate in Phase 2 successfully demonstrated these characteristics 
and proposed projects that aligned with existing organizational goals or projects.

To support the Phase 2 projects, the Sync for Genes program provided access to subject matter experts, resources, tools, and activities. Program activities included support for project planning and management via regularly scheduled meetings with each organization. Addressing feedback from Phase 1 regarding a request for more interaction among Sync for Genes participants, joint meetings were held every other month. These meetings provided participants with a forum for sharing challenges and lessons learned. Organizations were encouraged to participate in at least one HL7 FHIR Connectathon. In addition, participants had the opportunity to engage several subject matter experts who provided domain expertise (clinical genomics) and technical guidance (FHIR), and who facilitated the project team's interactions with HL7.

\section{Organization-Specific Projects}

Four organizations participated in Phase 2, each with their own individual projects that were driven by internal, independently funded initiatives that included the integration of genomic data into clinical care. This offered an opportunity to explore how current health IT standards support various needs to share genomic information. It is important to note that while progress on these initiatives was made during Phase 2, each organization continues to support these projects beyond the end of this program. The four use cases explored in Phase 2 were:

- The Utah Newborn Screening Program, operated by the Utah Department of Health, is developing the infrastructure to share raw genomic data with health care providers in real-time for optimal clinical care and outcomes for atrisk and vulnerable newborns.

- Weill Cornell Medicine is developing the ability to convert discrete genomic results to FHIR and pair these data with a physician-facing app providing supplemental knowledge of cancer genomic testing results.

- The National Marrow Donor Program (NMDP) is developing a tool to convert existing NGS-based human leukocyte antigen genotyping information to FHIR without losing information during the conversion process.

- Lehigh Valley Health Network made genomic data available in the electronic health record (EHR) and continues to test additional alerts tied to certain drug-gene pairs.

\section{Connectathon}

Three of the four organizations also participated in at least one HL7 FHIR Connectathon, which is an in-person event that provides an opportunity for technical developers to gain hands-on experience developing FHIR-based solutions and exchanging data through FHIR interfaces. ${ }^{22}$ Each connectathon is organized into tracks where attendees focus on developing or testing the standard against identified use cases. The Phase 2 organizations participated in a scenario that was designed to use FHIR to exchange genomic diagnostic reports. The connectathon offered organizations an opportunity to collaborate on one activity, applying and sharing the knowledge that they had been acquiring through Phase 2 , in a resource-rich envi- ronment surrounded by experts in FHIR. In providing their varying expertise and perspectives, the group identified gaps in the FHIR Clinical Genomics specification.

\section{Results}

The four organizations tested the ability of FHIR standard for trial use 3 (STU 3) and release 4 (R4) to support the various needs presented by each of their individual projects. Although progress was made in each organization's individual project during their participation in Phase 2, work on these projects continues as part of independently funded internal initiatives. Additionally, the refinement of the FHIR Clinical Genomics specification has continued within the HL7 Clinical Genomics Work Group, past the conclusion of Phase 2. The challenges and lessons learned using FHIR and the FHIR Clinical Genomics Implementation Guide were documented and shared with the HL7 Clinical Genomics Work Group. ${ }^{23}$ This feedback was provided verbally during working group meetings and asynchronously through the official HL7 issue tracking system. Several examples are summarized below.

The Utah Newborn Screening Program identified and tested potential FHIR resources for sending large, raw data files like variant call format (VCF) files. At first, the VCF file was created as a FHIR Binary resource element referenced in the FHIR Observation resource. ${ }^{24}$ However, this method would not support the inclusion of contextual information, such as author, procedure, or technique, which can be invaluable to the receiver of genomic data when those data are interpreted or analyzed further. As an alternative approach, the team explored using the attribute "valueAttachment" element in the Observation resource (FHIR STU 3). Although this resource element, typically used for images or reports in a PDF format, provides the additional contextual information, the data in this format cannot be easily parsed as it is not discrete. For purposes of this project, the FHIR R4 media resource was determined to be the best solution as it supports metadata that genomic files like VCF files require. However, there is uncertainty among implementers regarding how that resource will be able to support the exchange of the much larger files that are common in genomics.

Weill Cornell Medicine made progress in mapping workflows for their clinical genomics use cases to FHIR and in the development and testing of a modular application that can extract information from external knowledge sources to provide support for clinicians at the point-of-care. In preparation for the connectathon, this team found the ClinFHIR tool useful for visualizing the mapping of elements in the genomic diagnostic report to elements within FHIR resources and profiles. ${ }^{25}$ The team noted that the tool would have limited scalability because it requires manual creation of single documents.

NMDP successfully tested their FHIR conversion tool and plans to integrate it into a production environment via the NMDP Gateway. ${ }^{26}$ In the course of this work, NMDP found that there are insufficient provenance resources available for clinical genomics, which are essential to track the original source of the data and content of the genomic diagnostic report before conversion. 
During the course of the project, Lehigh Valley Health Network determined their enterprise was not ready to implement FHIR. However, they chose to continue participating in Phase 2 of this program. The organization made use of existing clinical infrastructure and its support for HL7 v2 messages to make genomic data available in the EHR. This team successfully tested a proof of concept that used alerts triggered by certain sensitivities or contraindications for three drug-gene pairs, which they are considering scaling to other types of care. The organization noted that they will continue to watch the development of FHIR and its use for exchanging genomic data.

Weill Cornell Medicine, NMDP, and the Utah Newborn Screening Program tested the ability of FHIR resource bundles to exchange genomic diagnostic reports that contain several results. Each team mapped their own diagnostic reports to FHIR and exchanged the FHIR renderings of their reports via a HAPI server that was available for use at the FHIR Connectathon 20, which took place in January 2019. ${ }^{27}$ The teams found that the FHIR Clinical Genomics Implementation Guide, the official specification detailing the use of FHIR for genomic data, did not provide sufficient documentation or examples to guide the use of resource bundles for this purpose. Specifically, within a genomic diagnostic report, there is the possibility that one result may reference another result within the same report, each of which may be referenced by separate FHIR resources and accessed via RESTful APIs if necessary. At the time of testing, the FHIR Clinical Genomics Implementation Guide did not provide clear guidance regarding the configuration and structure for that use case. The team encountered difficulty using HTTP POST when bundling portions of their diagnostic reports because the HAPI FHIR server used did not support that functionality with a collection bundle type. Over the course of their participation in connectathon activities, each organization successfully mapped their diagnostic reports to FHIR generating XML files and exchanged them as individual reports. Additionally, a concatenated version of the diagnostic reports was created to simulate a panel test in instances where results from various sources may need to be gathered and exchanged. The sample files of code for the genomic diagnostic reports and feedback provided to HL7 are available on the HL7 website. ${ }^{28-30}$

\section{Discussion}

In addition to the gaps identified during the course of leveraging FHIR to meet the goals of their individual projects and during connectathon activities, there were several lessons learned that could prove useful to health care organizations considering similar projects. These lessons learned gleaned from the testing and implementation of the health IT infrastructure that will make genomic data available at the point-of-care could be condensed into four categories: (1) FHIR proficiency, (2) developer support, (3) project scope, and (4) bridging health IT and genomic expertise.

\section{FHIR Proficiency}

Despite being embraced rapidly by the health IT and broader health care communities, FHIR is still a relatively new standard and one that is still under active development to suit the needs of use cases such as genomic medicine. The Phase 2 organizations' working knowledge of FHIR varied at the onset of the project. The Sync for Genes program provided a variety of resources and tools to bolster their understanding of the specification, including facilitating discussions with subject matter experts in both the FHIR and clinical genomics fields, as well as guiding them through FHIR documentation and tooling. The most impactful experience, however, was participation in an HL7 FHIR Connectathon, and the hands-on experience of applying a health IT standard to solve well-defined problems. The organizations found the dedicated time and face-to-face interaction with other developers and clinical genomics experts to be invaluable in gaining real-time, hands-on experience with the FHIR standard. For the connectathon, organizations were presented with a structured exercise created specifically for Phase 2 participants. Each step encountered challenges and identified slightly different gaps or issues within the FHIR Clinical Genomics specification. This exercise also highlighted the differences among the genomic diagnostic reports such as structural formatting, type of content, and level of detail.

An additional gap identified during Phase 2 was the need for foundational materials that support the consistent implementation of FHIR for clinical genomics use cases. As has been seen with other standards, consistent implementation of standards is needed to ensure interoperability across platforms and between organizations. In particular, interoperability will not be achieved if implementations diverge from standard guidance due to intentional localizations, unintended errors in implementation, or gaps, errors, or ambiguity in the specification. Throughout their projects, the organizations noted a need for additional guidance and clarification within the FHIR genomics documentation.

To help provide a robust foundation for the exchange of clinical genomic data, support was provided to help advance the development of a conceptual semantic information model for the clinical genomics domain. This type of information model provides details and definitions for clinical genomics concepts, how they are related to each other, and how they can be mapped to elements within FHIR resources and profiles. The work was focused on the analysis of existing HL7 standards and artifacts, including the Clinical Genomics Domain Analysis Model, which provides examples of clinical genomic use cases that can be supported by FHIR. This type of foundational work supports interoperability and alignment among the various HL7 standards, and can serve as a starting point for alignment with other standards by providing implementers with another central resource that connects different parts of the health IT implementation process. Continued development of the conceptual information model was successfully transferred to the HL7 Clinical Genomics Work Group, which is the custodial entity that oversees the development and maintenance of the FHIR Clinical Genomics specification and related artifacts. Development of the conceptual information model is not yet complete, but it represents an important resource in defining common semantics across the clinical genomics domain. 


\section{Developer Support}

The availability of FHIR-enabled health IT systems across the United States has been increasing. Approximately 96\% of hospitals that report information regarding their health IT systems to the Medicare EHR Incentive Program (MIPS) indicate that their health IT systems are both certified to the 2015 Edition Health IT Certification Criteria and are FHIR enabled. ${ }^{31,32}$ Although the saturation among MIPS eligible clinicians is lower, approximately $74 \%$, this is an increase from approximately 69\% since October $2018 .^{33}$ While this progress is evidence of the greater health IT community's embrace of the FHIR standard, it is one step in the greater journey of using FHIR in support of clinical genomics.

Currently, a majority of those health IT systems that are FHIR-enabled are referencing capabilities in FHIR DSTU2 (Second Draft Standard for Trial Use). The FHIR Clinical Genomics specification was initially developed using the FHIR DSTU2 specification, later upgraded to FHIR STU 3, and now leverages FHIR R4. The Phase 2 organizations experienced the mismatch between the FHIR version supported by enterprise tooling and that used by the latest version of the FHIR Clinical Genomics specification. The R4 version of the specification is more advanced and better supported the data used for testing, but implementing that version required the availability of health IT systems that support FHIR R4. There is an understandable lag as organizations allow standards to mature before fully implementing them in production settings, but the Phase 2 organizations found that even sandbox environments were not equipped to allow testing in FHIR R4. The conflict between what is necessary for the maturation of standards, which require extensive testing in production environments, and the priorities of health care organizations, which are often incentivized to wait for standards to mature prior to investing in their implementation within production systems, creates a dilemma for organizations involved in the development and adoption of standards. Although the health care industry may be ready to explore the routine integration of genomics at the point-of-care, this inconsistent support among different versions of health data standards has resulted in workarounds and additional needs for middleware when demonstrating even early stage proof of concepts. In Phase 2, one organization considered using middleware, creating additional implementation steps, to provide a temporary solution until FHIR R4 is natively available in their EHR. Another decided to move forward with pilot testing that leveraged current infrastructure and HL7 v2 messages, which accomplished the goal of delivering genomic data to the point-of-care but by using older technology. Two organizations noted they anticipated their EHR developers to provide modules in support of clinical genomics use cases in subsequent rounds of software updates; however, it is unclear what technology will be used in support of genomics or the extent of functionality and usability of the anticipated modules.

\section{Project Scope}

Organizational support for any enterprise-wide project is critical. The Phase 2 organizations submitted proposals that demonstrated organizational support as well as alignment with wider organizational strategy and ongoing efforts. Despite being at different phases of their respective projects, each was able to make advancements in the evaluation and/ or adoption of FHIR during the project period. The organizations collectively noted the importance of involving EHR developers, along with end-users such as clinicians, and administrative officers in advance.

As the possibilities of a reimagined health care delivery landscape in an era of precision medicine grow with the rapid technological advancements and scientific discoveries in genomics, it can be easy to get swept up in the excitement. To tether their work to the present, the organizations found that narrowly scoping projects was an effective way of demonstrating progress without being overwhelmed by the total effort that would be required for a full implementation of FHIR to support clinical genomics. The Sync for Genes program facilitated discussions regarding the use of mapping tools and techniques as starting points to further delineate their project scope, use cases or workflows. Budgets, the need for equipment, time, and other resources are also considerations that can limit project scope. Organizations found that leveraging existing infrastructure, approved strategic objectives, and drafting plans that took a systems approach were effective ways of securing internal buy-in for their projects.

\section{Bridging Health IT and Genomic Expertise}

The effective development, implementation, and use of health IT is an interdisciplinary venture requiring the active input from a variety of stakeholders to ensure interoperable, usable, and intuitive solutions that provide data and actionable knowledge to users. There is an opportunity to ensure that health data standards better meet the needs of precision medicine if health IT and genomic domain experts collaborate and understand their respective needs and limitations. There are several ways to participate in the FHIR and clinical genomics standards development process. ${ }^{23}$ The Phase 2 organizations found engaging with the standards community useful both in learning about the standards development process and in providing their own perspectives from each of their respective backgrounds. Several Phase 2 participants continued to actively participate in the working group past the end of the program. While the current HL7 Clinical Genomics Work Group has volunteer participants from a variety of backgrounds, organizations noted that the group may benefit from additional representation from genomic testing laboratories.

Organizations also found that participating in a cohortstyle project and having a forum for knowledge sharing as Phase 2 provided, particularly in a field that is so new, was invaluable. Insight regarding how other organizations plan to make clinical genomic data available at the point-of-care, the challenges others have encountered, and sharing some initial tactics helped these organizations better vet their own projects and organizational plans. This type of knowledge sharing can drive the market toward creation of health IT that supports clinical genomics beyond the point-of-care to other innovative uses of this data. Health IT clients and users should discuss expectations regarding functionality, capabilities, and ease of use with their technology developers and 
continue working in tandem throughout the process including through user testing phases. An interdisciplinary community that is inclusive of affected stakeholders is more likely to produce technology that will make this information rich, and sensitive data available to the right person at the right time, and with the proper controls.

\section{Conclusion}

The experience of the Sync for Genes Phase 2 organizations in testing FHIR for the exchange of clinical genomics data demonstrated the need for additional support for implementers in the form of documented guidance, knowledge sharing, training, and technical assistance. Incentives (including financial support) may be necessary to help encourage organizations to participate more actively in standards development, where early adoption is critical for maturation of standards but where the cost and risks of doing so are high. As organizations consider how they can enter the precision medicine era, these considerations and resources offer a starting point for identifying how to best apply genomics according to the mission of their organization. This project, in addition to strides in science and health IT, offers a glimpse of precision medicine.

Technology is a great enabler; however, it is only one piece of the equation necessary for the successful use of clinical genomics at the point-of-care. Accelerating precision medicine at the point-of-care will require advancements in many areas including integrating the evidence needed for clinicians to practice it. This evidence cannot be generated without a critical mass of relevant data. Sharing those data for research and facilitating its integration and aggregation will require use of relevant standards. Pilot testing supports the maturation of health data standards and can be a powerful demonstration of what is possible when clinical genomic data are electronically available and sharable. However, the effect of this rich information on medicine and science cannot be fully understood until it is available in production systems in health care and research environments.

\section{Clinical Relevance Statement}

Advancements in genomics have created new opportunities to leverage this rich source of information to enhance health research and ultimately change the way care is delivered. These data must be in an electronic, easy to parse format to ensure it can be appropriately and securely available for providers, consumers, and researchers. This project demonstrated the ability to use FHIR to support these capabilities, and that further testing, development, and adoption of this standard is needed so that it can better support the implementation of precision medicine.

\section{Multiple Choice Questions}

1. What is the purpose of Sync for Genes Phase 2?

a. To further develop and increase adoption of the FHIR genomic profiles in the clinical environment so that genomic data are available at the point-of-care and downstream for research.

b. To build a database that can be utilized by various stakeholders to house genomic data.

c. To develop standards for finding familial matches.

d. Develop genomic patient education materials.

Correct Answer: The correct answer is option a. Phase 2 supports significant follow-up steps to address these recommendations and enable faster adoption in clinical settings by determining what next steps were required of the FHIR Genomic profiles that were refined in phase 1. Sync for Genes Phase 2 is a collaboration between government and industry to further develop and increase adoption of the FHIR genomic profiles in the clinical environment, and build-out the standard to encompass other genomics-related activities.

2. What is the mission of the PMI?

a. To standardize how familial DNA matching can be utilized to treat patients.

b. To gather health data from one million people in the United States and make those data available to researchers.

c. To enable a new era of medicine through research and technology that empowers patients, researchers, and providers to work together toward the development of individualized treatments.

d. To advance the use of genomic data in clinical settings to improve patient care.

Correct Answer: The correct answer is option c. The Precision Medicine Task Force was established as a short-term group, convened to provide recommendations that support the President's Precision Medicine Initiative (PMI). ${ }^{1}$ The mission of the PMI is to enable a new era of medicine through research and technology that empowers patients, researchers, and providers to work together toward the development of individualized treatments. ONC is a key participant in the PMI, responsible for advancing data standards that support precision medicine, addressing relevant privacy policies, and advancing innovation in health IT. The task force will identify data standards and implementation specifications that make health IT data available to participants and researchers for precision medicine.

\section{Reference for Multiple Choice Questions}

1 Fact Sheet: President Obama's Precision Medicine Initiative. The White House President Barack Obama. Available at: https:// obamawhitehouse.archives.gov/the-press-office/2015/01/30/ fact-sheet-president-obama-s-precision-medicine-initiative. Published January 30, 2015. Accessed October 15, 2019

\section{Protection of Human and Animal Subjects}

There were no human and/or animal subjects included in this project.

Funding

None. 
Conflict of Interest

S.J.G. and T.Z.C. do not have any conflict of interest to report. R.R.F. was funded through U.S. Department of Health and Human Services Contract Number HHSP233201700054C with ESAC, Inc. to serve as technical director of this project via a consulting agreement. R.R.F. also serves as a co-chair of the HL7 Clinical Genomics Work Group, which authors and maintains the FHIR specification referenced in this article.

\section{Acknowledgments}

The authors would like to thank the organizations that participated in Sync for Genes Phase 2 including David E. Jones, Ph.D. and Nicole Ruiz-Schultz, Ph.D. from the Utah Newborn Screening Program; Michael Minear, M.S., CHCIO, CPHIMS, Senior Vice President and Chief Information Officer and Donald Levick, M.D., M.B.A., CPE, FHIMSS, Chief Medical Information Officer at the Lehigh Valley Health Network; Bob Milius, Ph.D., Principal Research Scientist at Center for International Blood and Marrow Transplant Research (CIBMTR); Sameer Malhotra, M.D., M.A., Assistant Professor of Healthcare Policy and Research at Weill Cornell Medicine, Attending Physician, Internal Medicine, New York-Presbyterian/Weill Cornell Medical Center. We would also like to thank Vera Rosenthal, MPH for copyediting support and Jon White, M.D., Administrative Chief of Staff for Research and Development Service at the Veterans Affairs in Salt Lake City Healthcare for his valuable advice on this document.

\section{References}

1 Fact Sheet: President Obama's Precision Medicine Initiative. The White House President Barack Obama. Available at: https:// obamawhitehouse.archives.gov/the-press-office/2015/01/30/factsheet-president-obama-s-precision-medicine-initiative. Published January 30, 2015. Accessed October 15, 2019

2 About. National Institutes of Health All of Us Research Program. Available at: https://allofus.nih.gov/about. Accessed August 21, 2019

3 Denny JC, Rutter JL, Goldstein DB, et al; All of Us Research Program Investigators. The "All of Us" Research Program. N Engl J Med 2019;381(07):668-676

$4 \mathrm{NCI}$ and the Precision Medicine Initiative ${ }^{\circledR}$. NIH National Cancer Institute. Available at: https://www.cancer.gov/research/areas/ treatment/pmi-oncology. Updated July 24, 2017. Accessed on October 15, 2019

5 precisionFDA. U.S. Food and Drug Administration. Available at: https://precision.fda.gov/. Accessed October 15, 2019

6 Precision Medicine. HealthIT.gov. Available at: https://healthit. gov/topic/precision-medicine. Published July 3, 2019. Accessed October 15, 2019

7 HHS Awards \$21 Million to Support Health Center Participation in NIH's All of Us Research Program. HHS.gov. Available at: https:// www.hhs.gov/about/news/2018/09/11/hhs-awards-21-millionsupport-health-center-participation-nih-research-program. html. Published September 11, 2018 Accessed October 15, 2019

8 21st Century Cures Act. Washington, DC. December 13, 2016. 114th Congress. 42 USC 201 Note. Available at: https://www. congress.gov/114/plaws/publ255/PLAW-114publ255.pdf. Accessed August 21, 2019

9 What is Precision Medicine? U.S. National Library of Medicine. Available at: https://ghr.nlm.nih.gov/primer/precisionmedicine/ definition. Published April 2015. Updated January 7, 2020. Accessed January 17, 2020

10 Precision Medicine in Cancer Treatment. NIH National Cancer Institute. Available at: https://www.cancer.gov/about-cancer/ treatment/types/precision-medicine. Updated October 3, 2017. Accessed August 21, 2019

11 Lander ES. Initial impact of the sequencing of the human genome. Nature 2011;470(7333):187-197

12 Green ED, Guyer MS; National Human Genome Research Institute. Charting a course for genomic medicine from base pairs to bedside. Nature 2011;470(7333):204-213

13 Kennell TI Jr, Willig JH, Cimino JJ. Clinical informatics researcher's desiderata for the data content of the next generation electronic health record. Appl Clin Inform 2017;8(04):1159-1172

14 Rasmussen LV, Overby CL, Connolly J, et al. Practical considerations for implementing genomic information resources. Experiences from eMERGE and CSER. Appl Clin Inform 2016;7(03): 870-882

15 Mate S, Kampf M, Rödle W, et al. Pan-European data harmonization for biobanks in ADOPT BBMRI-ERIC. Appl Clin Inform 2019; 10(04):679-692

16 Welcome to $\mathrm{FHIR}^{\circledR}$. HL7 ${ }^{\circledR}$ International. Available at: http://hl7. org/fhir. Published November 1, 2019. Accessed January 9, 2020

17 Matney SA, Heale B, Hasley S, et al. Lessons learned in creating interoperable Fast Healthcare Interoperability Resources profiles for large-scale public health programs. Appl Clin Inform 2019;10 (01):87-95

18 Gordon WJ, Baronas J, Lane WJ. A FHIR human leukocyte antigen (HLA) interface for platelet transfusion support. Appl Clin Inform 2017;8(02):603-611

19 Maranhão PA, Bacelar-Silva GM, Ferreira DNG, Calhau C, VieiraMarques P, Cruz-Correia RJ. Nutrigenomic information in the openEHR data set. Appl Clin Inform 2018;9(01):221-231

20 Zayas Cabán T, Okubo T, Chaney K. These Genes are on FHIR! Advances in standardizing genomics through HL7® FHIR®. Health IT Buzz. Available at: https://www.healthit.gov/buzzblog/precision-medicine/genes-fhir-advances-standardizing-genomics-hl7-fhir. Published November 28, 2017. Accessed August 21, 2019

21 Alterovitz G, Brown J, Chan M, et al. Enabling Clinical Genomics for Precision Medicine Via HL7® Fast Healthcare Interoperability Resources ${ }^{\circledR}$. Available at: https://healthit.gov/syncforgenes-phase1report. Published November 28, 2017. Accessed August 21, 2019.

22 HL7® FHIR® Connectathons. HL7 ${ }^{\circledR}$ International. Available at: http://www.hl7.org/events/fhir-connectathon/index.cfm. Accessed August 21, 2019

23 Clinical Genomics. HL7 ${ }^{\circledR}$ International. Available at: http://www. hl7.org/Special/committees/clingenomics/overview.cfm. Updated September 21, 2016. Accessed January 10, 2020

24 2.3.5 Resource Binary - Content. HL7 ${ }^{\circledR}$ FHIR ${ }^{\circledR}$ Release 4. Available at: https://www.hl7.org/fhir/binary.html. Published November 1, 2019. Accessed January 10, 2020

25 ClinFHIR Launcher. Available at: http://clinfhir.com/. Published August 3, 2015. Accessed January 10, 2020

26 NMDP FHIR Conversion Tool. GitHub. Available at: https://github. com/nmdp-bioinformatics/hml-fhir-app. Accessed August 21, 2019

27201901 Clinical Genomics. HL7 ${ }^{\circledR}$ International. Available at: https:// wiki.hl7.org/201901_Clinical_Genomics. Updated March 20, 2019. Accessed August 21, 2019

28 File: Final S4GP2 Connectathon Utah NBS.zip. HL7 ${ }^{\circledR}$ International. Available at: https://wiki.hl7.org/File:Final_S4GP2_Connectathon_Utah_NBS.zip. Published March 20, 2019. Accessed January 10, 2020

29 File: Completed Cornell CaT Files.zip. HL ${ }^{\circledR}$ International. Available at: https://wiki.hl7.org/index.php?title=File:Completed_Cornell_ CaT_Files.zip. Published March 20, 2019. Accessed January 10, 2020

30 File: NMDP Original and Final Files.zip. HL $7^{\circledR}$ International. Available at: http://wiki.hl7.org/index.php?title=File:NMDP_Original_and_ 
302 Sync for Genes: Genomics at the Point-of-Care Garcia et al.

Final_Files.zip. Published March 20, 2019. Accessed January 10, 2020

31 API Information for 2015 Edition Products. HealthIT.gov. Available at: https://chpl.healthit.gov/\#/collections/api-documentation. Updated December 31, 2019. Accessed January 10, 2020

32 EHR Products Used for Meaningful Use Attestation. HealthIT.gov. Available at: https://dashboard.healthit.gov/datadashboard/doc- umentation/ehr-products-mu-attestation-data-documentation. php. Updated January 10, 2019. Accessed January 10, 2020

33 Posnack S, Barker W. Heat Wave: The U.S. is Poised to Catch FHIR in 2019. Health IT Buzz. Available at: https://www.healthit.gov/ buzz-blog/interoperability/heat-wave-the-u-s-is-poised-tocatch-fhir-in-2019. Published October 1, 2018. Accessed August 21, 2019 